\title{
LA PLANIFICACIÓN URBANA DE LIMA-CALLAO 1949-2013: DEL URBANISMO FUNCIONALISTA A LA PLANIFICACIÓN DEL DESARROLLO URBANO SOSTENIBLE
}

\section{Rodolfo Francisco Castillo Garcia}

\section{Resumen}

El objetivo del presente artículo es hacer algunas reflexiones acerca de la evolución de la planificación urbana del Área Metropolitana Lima - Callao (Perú) en el periodo 1949 - 2013, y proponer que la formulación del Plan de Desarrollo Metropolitano Lima - Callao 2013 - 2035 se haga con el enfoque emergente de la planificación del desarrollo urbano sostenible.

Palabras Clave: Planificación urbana, planificación del desarrollo urbano sostenible.

\section{Abstract}

The aim of this article is to offer some reflections about the evolution of urban planning in the Metropolitan Area of Lima - Callao (Peru) in the period 19492013, and proposes that the Metropolitan Development Plan of Lima - Callao 2013 - 2035 should be done with the emerging approach of the sustainable urban development planning.

Key words: Urban planning, sustainable urban development planning. 


\section{EVOLUCIÓN DE LA PLANIFICA- CIÓN URBANA CONTEMPORÁNEA Y DE LA PLANIFICACIÓN URBANA DE LIMA-CALLAO 1949-2013}

En 1990, Lima - Callao constituian una Metrópolis, con una población aproximada de 6500,000 habitantes. Y hoy, 23 años después, la realidad ha cambiado significativamente. Lima-Callao posee una población aproximada de 9`500,000 habitantes ${ }^{1}$ y está en los umbrales históricos de convertirse en una Megalópolis. El término Megalópolis se aplica a la conurbación de áreas metropolitanas y/o a la superposición de sus áreas de influencia, haciendo referencia a un sistema urbano que cuenta con una población igual o superior a los 10 millones de habitantes.

Como 23 años después no sólo la realidad urbana de Lima - Callao es diferente, también tiene que serlo el enfoque de planificación urbana para abordarla y transformarla. De hecho, la planificación urbana contemporánea ha evolucionado, desde las primeras décadas del siglo XX, de una planificación urbana normativa, disciplina ligada al urbanismo funcionalista, y orientada al acondicionamiento físico de los centros urbanos y a la regulación de los usos del suelo urbano; hacia una planificación del desarrollo urbano sostenible, en el siglo XXI, como una interdisciplina ligada a la sostenibilidad urbana.

\subsection{Evolución de la Planificación Urbana Contemporánea}

En Europa y América, desde las primeras décadas del siglo $\mathrm{XX}$ hasta hoy, la planificación urbana contemporánea ha sido aplicada y se aplica con diferentes enfoques teórico - metodológicos, como los que se identifican a continuación.

La planificación urbana normativa (o planificación urbana tradicional), originada en las primeras décadas del siglo $\mathrm{XX}$, se caracteriza por su énfasis en la formulación de planes fundamentalmente fisico - espaciales, con un enfoque orientado a la oferta urbana, y por su intención de regulación normativa de los usos del suelo ${ }^{2}$.

Ante las limitaciones estructurales de la planificación urbana normativa a inicios de los años 80 del siglo $\mathrm{XX}$, así como su falta de flexibilidad y eficacia ante la dinámica de la urbe y su débil conocimiento de la evolución de los procesos sociales, se hizo necesario que a fines de los años 80 se generaran otros enfoques de planificación urbana.

Así, se planteó la planificación urbana estratégica como una forma ligada a la búsqueda de cambios cualitativos en los procesos de desarrollo de la ciudad, y a la implementación de proyectos estratégicos de desarrollo urbano. No obstante, ello sólo habría significado impactos en sectores focalizados de la ciudad, generado segregación social urbana, y no necesariamente involucraría y/o beneficiaría a la mayoría de estratos sociales que viven en ella.

Y también se propuso y desarroló la planificación urbana ambiental como una forma de incorporar la dimensión ambiental en el proceso 
planificador de las ciudades. Empero, ésta no resuelve la incorporación de temas como crecimiento competitivo, equidad social urbana y gobernabilidad.

En los inicios del siglo XXI, se comenzó a hablar de la gobernanza (o gobernabilidad) como una forma de gestión urbana que privilegia la acción de los actores sociales en el desarrollo de las ciudades, lo cual obliga a repensar el tema de las modalidades actuales de gestión urbana y ambiental. Empero, la gobernanza no ha demostrado hasta ahora ser suficiente como sustitución de la planificación urbana moderna, sino sólo como su complementación.

Actualmente, se habla de la planificación del desarrollo urbano sostenible que postula incorporar la sustentabilidad ambiental, la competitividad económica y la equidad social en el desarrollo de la ciudad. Empero, aún no ha sido asimilada en términos normativos en la mayoría de los países, y no se ha establecido su aplicación metodológica en los planes urbanos, salvo aproximaciones valiosas.

\subsection{Evolución de la Planificación} Urbana de Lima-Callao 1949-2013

En este contexto, el desarrollo de los enfoques en la planificación urbana contemporánea en sus sucesivos estados del arte influyó en la planificación urbana de Lima - Callao, de la siguiente manera:

- En el periodo 1949-1968 los primeros planes urbanos de Lima - Callao se referian a aspectos de zonificación urbana y esquemas viales; los cuales reflejaban la influencia del urbanismo funcionalista, fundamentalmente físico - espacial y esteticista: Plan Piloto de Lima, de 1949, y Plan Regulador, de 1956.

- En el periodo 1969-1987, la planificación urbana en el Perú evolucionó desde planes urbanos físico - espaciales hacia planes urbanos más complejos e interdisciplinarios; perfeccionados con un mejor conocimiento de los comportamientos reales del desarrollo urbano, y con políticas urbanas más operativas e instrumentos técnicos, consolidándose como planificación urbana normativa en Lima - Callao: Plan de Desarrollo Metropolitano Lima Callao 1968-1980 - PLANDEMET.

- En el periodo 1988-1996, la planificación urbana normativa en Lima- Callao devino en la planificación urbana ambiental, con planes urbanos que incorporaban de modo coherente la dimensión ambiental en sus estudios y previsiones; complementados en aspectos de gestión, y considerando los mecanismos de mercado: Plan de Desarrollo Metropolitano Lima Callao 1990-2010 - PLANMET, Plan Urbano Director de la Provincia Constitucional del Callao 1995-2010, y Plan de Desarrollo de la Costa Verde de Lima Metropolitana 1995-2010.

- En el periodo 1997-2011, la planificación urbana ambiental en el Perú se complejizó al incorporar elemen- 
tos innovadores de la planificación urbana estratégica, con planes urbanos se realizaron ligados a la búsqueda de cambios cualitativos en los procesos de desarrollo de las ciudades con la implementación de acciones estratégicas de desarrollo urbano y considerando mejor los mecanismos del mercado, la globalización y la competitividad. Sin embargo, en Lima-Callao en este periodo hubo un decaimiento de la planificación urbana debido a una indefinición metodológica en el IMP para asumir la planificación urbana estratégica y a una confusión entre planificación integral y planificación urbana: Plan Integral de Desarrollo Metropolitano de Mediano Plazo 1998-2002 (inconcluso).

- En el periodo 2012-2013, en LimaCallao se plantean las primeras aproximaciones teóricas y se concretan las primeras aplicaciones metodológicas y operativas de la planificación del desarrollo urbano sostenible. No obstante, en la actualidad este enfoque de planificación emergente todavía no ha sido asimilado normativamente y no se ha dispuesto su aplicación metodológica y operativa en los planes urbanos: Plan Especifico de Villa San Antonio y Quebradas Canto Grande y Media Luna 2012-2025.

- Cabe señalar que la planificación urbana en Lima - Callao no ha experimentado un cambio radical de un enfoque teórico por otro, sino que se ha ido complementando en un enfoque integrador pragmático implicito, que ha permitido arribar hasta hoy a una planificación urbana ambiental estratégica. En este contexto, se requiere un enfoque integrador emergente explícito que recoja los aportes de los diferentes enfoques de planificación urbana moderna y supere sus respectivas limitaciones, para lograr una planificación urbana más viable, eficaz, eficiente y socialmente legitimada. Dicho enfoque emergente es la planificación del desarrollo urbano sostenible.

\section{PLANES URBANOS DE LIMA - CALLAO EN EL PERIODO 1949-2013}

Los planes urbanos más importantes de Lima - Callao entre 1949 y el 2013, que constituyen hitos en la evolución de la planificación urbana de Lima - Callao, son los siguientes:

\subsection{Plan Piloto de Lima de 1949}

El Plan Piloto de Lima de 1949, se constituyó en la primera etapa del que después se plasmó como el Plan Regulador de Lima de 1956. Fue elaborado por la Oficina Nacional de Planeamiento y Urbanismo, y aprobado por Resolución Suprema $\mathbf{N}^{\circ} 258$ del Ministerio de Fomento y Obras Públicas, en Septiembre de $1949^{3}$.

Constituye un ejemplo de la influencia del urbanismo funcionalista de principios del siglo XX, y de planificación urbana normativa, incidiendo básicamente en los aspectos físicofuncionales y de diseño urbano. 


\subsection{Plan de Desarrollo Metropolitano de Lima Callao 1968-1980}

El Plan de Desarrollo Metropolitano Lima Callao 1968-1980 - PLANDEMET fue formulado por la Oficina Nacional de Planeamiento y Urbanismo - ONPU, entre 1965 y 1968; en convenio con la Municipalidad de Lima, durante la administración del Alcalde Luis Bedoya Reyes.

Es el ejemplo más significativo, complejo e interdisciplinario de planificación urbana normativa de nivel metropolitano entre 1947 y 1968. En él también se incorporó temas de densificación y expansión urbana, vivienda y renovación urbana, áreas industriales, vialidad $\mathrm{y}$ transporte, equipamiento urbano, recreación, normativa urbana, etc. No obstante, la dimensión ambiental no se desarrolló explícitamente, sino vinculada al tema de recreación y áreas verdes.

\subsection{Plan de Estructuración Urbana Metropolitana de Lima 1986-1996}

El Plan de Estructuración Urbana Metropolitana de Lima 1986-1996 fue elaborado por la Municipalidad de Lima Metropolitana. Fue aprobado mediante el Acuerdo de Concejo $\mathbf{N}^{\circ}$ 270-1986, durante la administración del Alcalde Alfonso Barrantes Lingán.

Fue una experiencia de planificación urbana normativa. No obstante, constituyó un plan piloto que no llegó a constituir un plan de desarrollo metropolitano propiamente dicho. La dimensión ambiental y de seguridad fisica ante desastres no se desarrolló como tal, sino puntualmente en algunas politicas de suelo y de infraestructura de servicios.
2.4. Plan de Desarrollo Metropolitano Lima-Callao - PLANMET 1990-2010

El Plan de Desarrollo Metropolitano Lima - Callao 1990-2010 - PLANMET fue formulado entre 1987 y 1989 por el Proyecto Especial PLANMET de la Municipalidad de Lima, y aprobado en 1989 mediante el Acuerdo de Concejo $\mathbf{N}^{\circ}$ 287-1989, durante la administración del Alcalde Jorge Del Castillo.

Fue actualizado y publicado por el Instituto Metropolitano de Planificación - IMP de la Municipalidad de Lima Metropolitana, en cumplimiento del Acuerdo de Concejo $\mathbf{N}^{\circ}$ 167-1991, y puesto en vigencia por Decreto de Alcaldía $\mathbf{N}^{\circ}$ 127-1992, durante la administración del Alcalde Ricardo Belmont Cassinelli. Fue ratificado por la Ordenanza No 099-1996, durante la gestión del Alcalde Alberto Andrade Carmona. Y ha sido prorrogado en su vigencia mediante las Ordenanzas Municipales $\mathrm{N}^{\circ} 1505-2011, \quad \mathrm{~N}^{\circ}$ 1604-2012 y $\mathbf{N}^{\circ}$ 1702-2013 por la actual gestión de la Alcaldesa Susana Villarán De la Puente.

Es un hito importante de planificación urbana ambiental en la planificación urbana metropolitana, ya que incorpora pioneramente, aunque sin llegar a integrar la dimensión ambiental en un plan urbano de esta jerarquía, definiendo entre sus objetivos específicos el ordenamiento ambiental metropolitano, politicas generales de medio ambiente $y$ seguridad ante desastres; y una propuesta general de recuperación ambiental. 
Con el PLANMET, la planificación urbana normativa en Lima-Callao empezó a transformarse en planificación urbana ambiental, aunque metodológicamente la incorporación coherente de la dimensión ambiental en los planes urbanos demandó un proceso de maduración posterior.

Otro aporte fundamental del PLANMET es que propuso la creación del Instituto Metropolitano de Planificación - IMP como organismo permanente de la Municipalidad de Lima Metropolitana, para que se encargue de la planificación del desarrollo local en la Provincia de Lima; así como del seguimiento, evaluación, retroalimentación y actualización del PLANMET. Así, se generó un proceso permanente de planificación desde la creación del IMP en 1991 hasta 1996, que se expresó en programas, normas, informes técnicos, planes urbanos distritales y especiales, convenios de cooperación, etc.; dirigidos a orientar y racionalizar la toma de decisiones vinculada con el desarrollo urbano metropolitano sostenible.

Planteó la estructuración del Sistema Vial Metropolitano mediante una red jerarquizada para procurar la mejor interrelación entre las Grandes Áreas Urbanas de la Metrópoli, comprendiendo los 5 Anillos Viales de Desconcentración y las Rutas del Sistema de Transporte Rápido Masivo.

Constituye un hito importante en la planificación urbana de Lima - Callao, ya que es el único plan metropolitano que contiene un Programa de Inversiones, a partir del cual se generó un pro- ceso permanente de planificación - preinversión, que se expresa en proyectos, acciones y obras, que constituyen los mecanismos más eficaces para encaminar el desarrollo urbano hacia los objetivos que el Plan señala.

Dicho proceso de planificación con énfasis económico - financiero, a partir de vincular la planificación, la preinversión y las obras, posibilitó al IMP la elaboración de más de 200 estudios específicos entre 1991 y 1996, muchos de los cuales permitieron a las últimas gestiones municipales, ejecutar obras en beneficio de la ciudad, como las siguientes, entre otras:

- Construcción de la Av. Universitaria, que forma parte del Anillo Vial de Desconcentración $N^{\circ} 3$.

- Construcción del Intercambio Vial Norte Av. Universitaria - Carretera Panamericana Norte, que es parte del Anillo Vial de Desconcentración $\mathrm{N}^{\circ} 3$.

- Construcción del Intercambio Vial Sur Av. Javier Prado - Vía de Evitamiento - Carretera Panamericana Sur, que forma parte de los Anillos Viales de Desconcentración $\mathrm{N}^{\circ} 3 \mathrm{y}$ $\mathrm{N}^{\circ} 5$.

- Construcción del Intercambio Vial Este Av. Riva Agüero - Av. Próceres de la Independencia, que forma parte del Anillo Vial de Desconcentración $\mathrm{N}^{\circ} 2$.

- Mejoramiento de Paso a Desnivel Av. Primavera - Carretera Panamericana Sur, que forma parte del Anillo Vial de Desconcentración $N^{\circ} 4$.

- Revalorización de la Plaza de Armas, Plaza San Martín y Parque 
Universitario, que forman parte del Centro Histórico de Lima.

- Mejoramiento del Trébol de Caquetá, que forma parte del Anillo Vial de Desconcentración $\mathrm{N}^{\circ} 5$.

- Construcción del Paso a Desnivel Av. Universitaria - Av. Perú, que forma parte del Anillo Vial de Desconcentración $\mathrm{N}^{\circ} 3$.

- Construcción de los Intercambios Viales Av. Universitaria - Av. Argentina, Av. Universitaria - Av. O. R. Benavides (ex Av. Colonial) y Av. Universitaria - Av. Venezuela, que forman parte del Anillo Vial de Desconcentración $\mathrm{N}^{\circ} 3$.

- Construcción del Paso a Desnivel Av. El Derby - Av. San Borja Norte.

- Construcción de la Vía Expresa e Intercambios Viales de la Av. Javier Prado, que forma parte del Anillo Vial de Desconcentración N ${ }^{\circ} 3$.

- Construcción de la Vía Expresa Av. Grau, que forma parte del Anillo Vial de Desconcentración $N^{\circ} 1$.

- Promoción de terminales terrestres interprovinciales en Lima Norte, Lima Sur y Lima Este.

\subsection{Plan Urbano Director de la Provincia Constitucional del Callao 1995-2010}

El Plan Urbano Director de la Provincia Constitucional del Callao 1995-2010, fue elaborado por el IMP de la Municipalidad de Lima, en virtud a un convenio entre la Corporación de Desarrollo del Callao, la Municipalidad Provincial del Callao y el IMP. Fue aprobado por la Municipalidad Provincial del Callao por Ordenanza Municipal $\mathbf{N}^{\circ}$ 018-
1995, y actualmente está en plena vigencia, mediante las Ordenanzas Municipales $\mathrm{N}^{\circ}$ 003-2011 y $\mathrm{N}^{\circ} 010$ 2011.

Es un hito importante de planificación urbana ambiental, ya que se formuló de acuerdo a los lineamientos del PLANMET, integrando coherentemente la dimensión ambiental en un plan urbano. Con este plan, la planificación urbana normativa en Lima - Callao se consolida definitivamente como planificación urbana ambiental.

Otro aporte del Plan Urbano del Callao es que permitió vincular la planificación, la preinversión y las obras a partir de sus previsiones, que se expresan en proyectos, acciones y obras estratégicas de desarrollo provincial y urbano. Algunos ejemplos son, entre otros:

- Construcción del Intercambio Vial Av. Elmer Faucett - Av. O. R. Benavides (ex-Colonial), emprendido por CORDECALLAO, hoy Gobierno Regional del Callao.

- Ensanche de la Av. Elmer Faucett, entre el Aeropuerto Internacional Jorge Chávez y la Av. Argentina, y la construcción del Corredor Vial Av. Venezuela; ejecutados por la Municipalidad del Callao; lo que ha mejorado la operación de las vías y el acceso al terminal aeroportuario más importante del país.

- Rehabilitación de la Av. República de Panamá, la construcción del Estadio Miguel Grau, del Coliseo Cerrado y de la Piscina Olímpica en el Parque Yahuar Huaca, así como las obras de rehabilitación de las 
Av. Argentina y Néstor Gambetta; realizados por CORDELICA, hoy Gobierno Regional del Callao.

- Rehabilitación y reordenamiento de las Av. Sáenz Peña y Buenos Aires, y el reordenamiento del comercio ambulatorio en el centro del Callao, emprendidos por la Municipalidad del Callao; lo que ha mejorado las condiciones urbano - ambientales del centro comercial del Callao.

- Construcción de la Autopista Callao - Canta, emprendida por el ex-Ministerio de Transportes, Comunicaciones, Vivienda y Construcción.

- Modernización y ampliación de las instalaciones del Aeropuerto Internacional Jorge Chávez, a través de la concesión a un operador internacional.

- Construcción del Intercambio Vial de la Av. Guardia Chalaca: Ovalo Saloom y Av. Sáenz Peña; por CTAR Callao, hoy Gobierno Regional del Callao.

- Elaboración de los estudios de ingeniería listos para la licitación de las obras de la Vía Costa Verde en el tramo del Callao: Intercambio Vial Av. Faucett - Av. Venezuela, y de las obras de revalorización del Centro Histórico del Callao.

- Elaboración del estudio de factibilidad técnica del Intercambio Vial Av. Faucett - Av. Tomás Valle.

- Ejecución de diversos programas de vivienda del Gobierno Central en la Urb. Antonia Moreno de Cáceres, Ciudad Pachacútec, y en los asentamientos humanos San
Judas Tadeo y Puerto Nuevo, etc. Aunque en Ciudad Pachacútec, la reubicación de 35,000 habitantes hecha por el Gobierno Central en el año 2000, sin haberse realizado previamente las obras de habilitación urbana necesarias, rebasó las previsiones de este Plan, generando un problema social, urbano y ambiental considerable.

2.6. Plan Maestro de Desarrollo de la Costa Verde de Lima Metropolitana 1995-2010

El Plan Maestro de Desarrollo de la Costa Verde de Lima Metropolitana 1995-2010 constituye un plan urbano especial, que fue elaborado por el IMP de la Municipalidad de Lima, en convenio con la Autoridad del Proyecto Costa Verde - APCV, y aprobado por Acuerdo de la APCV N 79-1995.

Es otro ejemplo valioso de planificación urbana ambiental del borde costero, ya que se formuló de acuerdo a los lineamientos del PLANMET, e integrando la dimensión ambiental a un plan urbano especial, constituyó un aporte al desarrollo del corredor ribereño de la Costa Verde de Lima.

En cuanto a las limitaciones institucionales del Plan Maestro, cabe señalar que no se diseñó los instrumentos técnicos suficientes orientados a la consolidación institucional de la Autoridad del Proyecto Costa Verde - APCV, y a su implementación técnica con fines de gestión y seguimiento sistemático y permanente del Plan, a través de una Secretaría Técnica propia. 
Asimismo, diseñó mecanismos para la participación de la inversión privada en proyectos turísticos y de servicios, pero priorizó como estrategia la ejecución de los proyectos de inversión estructurantes sobre la base de la inversión pública, lo cual ha generado obras fundamentalmente en vialidad. No obstante, la inversión privada se ha sumado de manera conservadora a través de estudios de preinversión y de no muchos casos de inversión efectiva.

La implementación de este Plan Maestro de Desarrollo de la Costa Verde se vio dificultada fundamentalmente entre 1995 y el 2005 por:

- La demora en implementar las normas que regulan el desarrollo de dicho corredor ribereño; definir la personería jurídica y la estructura orgánica de la APCV; así como los procedimientos para la gestión y realización de los proyectos de inversión privada, pública o mixta para su desarrollo integral. Esta tarea recién se implementó 10 años después de aprobado el Plan Maestro, mediante la Ordenanza Municipal $\mathbf{N}^{\circ}$ 750-2005 de la Municipalidad de Lima.

- La demora en la ejecución de las obras de saneamiento ambiental del litoral metropolitano de Lima Callao, orientadas a contrarrestar la contaminación de las aguas marinas por la presencia del Colector Costanero de aguas servidas, que se desactivó después del 2005.

- La APCV no respetó las secciones viales normativas que el Plan
Maestro de 1995 estableció para el Circuito de Playas de la Costa Verde (3 carriles de ida y 3 carriles de vuelta, además de una vía de servicio a las playas), dando la conformidad a proyectos incompatibles con el Plan Maestro. Estos derivaron en construcciones antirreglamentarias que ocupan sectores de playa (que sólo deberían ser para bañistas y tablistas) o invaden el derecho de vía normativo del Circuito de Playas en el sector del Distrito de Barranco, violando flagrantemente las previsiones del Plan Maestro.

\subsection{Plan Integral de Desarrollo Metropolitano de Mediano Plazo 1998-2002}

La formulación del Plan Integral de Desarrollo Metropolitano de Mediano Plazo 1998-2002, fue dispuesta por la Ordenanza Municipal $\mathbf{N}^{\circ}$ 0991996, durante la gestión del Alcalde Alberto Andrade Carmona, para que el IMP lo elaborara sobre la base de Planes Integrales Distritales. Este Plan Integral debió ser elaborado entre 1996 y 1998, para ser aprobado en Agosto de 1998, pero nunca fue culminado ni aprobado.

Fue un intento fallido e inconcluso de planificación integral de desarrollo de nivel metropolitano, que debía incluir en sus estudios, aspectos económicos, sociales, culturales e institucionales, así como objetivos, estrategias, políticas y propuestas de desarrollo. Pero además, pretendía incluir aspectos urbanos ya previstos en el PLANMET Lima - Callao vigente, con 
la finalidad de reemplazarlo; cuando en realidad eran planes de diferente naturaleza y con diferente ámbito territorial, generando una confusión conceptual entre la planificación integral del desarrollo y la planificación urbana.

\subsection{Plan de Desarrollo de la Región} Lima Metropolitana 2003-2006

El Plan de Desarrollo de la Región Lima Metropolitana 2003-2006 apuntaba a constituirse en el primer esfuerzo de conocimiento de la realidad de la ciudad capital considerada como región, orientado a trazar un rumbo posible a mediano plazo, así como sentar las bases para el establecimiento de políticas de desarrollo sostenible a largo plazo.

Fue un esfuerzo de planificación regional estratégica. Sin embargo, no tenía un claro énfasis económico productivo y social para diferenciarlo del futuro Plan de Acondicionamiento Territorial y Desarrollo Urbano de Lima; ya que la mayoría de sus proyectos regionales estratégicos aún eran proyectos territoriales, urbanos o ambientales urbanos.

Por otro lado, no consideró como ámbito de análisis al área que se denominó Macro Sistema Lima, que incluye a las Micro - Regiones Compensatorias de Huacho - Barranca al norte, y Cañete - Chincha - Pisco al sur de Lima Metropolitana; como una aproximación analítica al concepto de la Región Metropolitana Nacional, planteado en los años 70 por el PLANDEMET.

2.9. Plan Regional de Desarrollo Concertado de Lima 2012-2025
El Plan Regional de Desarrollo Concertado de Lima 2012-2025 ha sido aprobado por la Municipalidad Metropolitana de Lima mediante la Ordenanza Municipal $\mathrm{N}^{\circ}$ 16592013. Es un esfuerzo apreciado de planificación regional estratégica; empero, no tiene un claro énfasis económico - productivo y social, sino mas bien urbanístico, y la mayoría de sus proyectos regionales estratégicos son proyectos territoriales, urbanos o ambientales.

Los temas prioritarios de Plan Regional de Desarrollo Concertado 2012-2035 son: seguridad ciudadana; movilidad, transporte y vialidad; crecimiento urbano; agua, saneamiento y vivienda; educación y salud; medioambiente, riesgo y vulnerabilidad; espacios públicos, áreas verdes y recreación; cultura y desarrollo; economía, comercio, producción y servicios; y pobreza urbana.

\section{LA PLANIFICACIÓN DEL DESA- RROLLO URBANO SOSTENIBLE Y EL PLAN DE DESARROLLO METROPO- LITANO LIMA CALLAO 2013-2035}

Es necesario encontrar formas innovadoras de planificación urbana y contar con instrumentos prácticos, integrados y holísticos que respondan a los procesos de descentralización y a la demanda de mayor participación de la sociedad civil; así como elaborar enfoques teóricos, metodológicos y prácticos para inducir planificadamente los cambios necesarios para la transformación de las ciudades en el futuro ${ }^{4}$. 
Por tanto, se requieren nuevos enfoques teóricos y/o metodológicos de planificación urbana contemporánea para superar las actuales limitaciones estructurales de ésta, a fin de constituir una planificación urbana más viable, eficaz, eficiente y socialmente legitimada en el tratamiento, previsión y promoción del desarrollo urbano sostenible de las ciudades.

Asimismo, se considera que la utilización conjunta de enfoques de planificación urbana complementarios puede ser una manera de integrar diferentes actividades y asegurar una finalidad y una dirección común ${ }^{5}$.

En esta perspectiva y en el marco de la sostenibilidad urbana, la planificación del desarrollo urbano sostenible es un enfoque integrador emergente explícito que recoge aportes de los diferentes enfoques de planificación urbana moderna y supera sus respectivas limitaciones para procurar el tratamiento, previsión y promoción del desarrollo urbano sostenible de las ciudades.

Dicho enfoque integrador emergente explícito en la planificación urbana moderna, se puede sintetizar en términos de planificación - regulación acción - gestión - sustentabilidad, en la medida en que debe ser una integración entre elementos sustantivos, $\mathrm{de}^{6}$ :

- La planificación urbana normativa (planificación - regulación), que proporciona instrumentos para regular un desarrollo físico ordenado y atractivo de la ciudad.

- La planificación urbana estratégica (planificación - acción), que procura el crecimiento urbano competitivo, poniendo los medios para alcanzar la prosperidad económica urbana.

- La gobernanza (planificación - gestión), que procura lograr acuerdos para promover la equidad social en el espacio urbano y el equilibrio de los intereses y expectativas de los actores económicos y sociales de la comunidad urbana.

\section{- La planificación urbana ambien-} tal (planificación - sustentabilidad), que incorpora la sustentabilidad ambiental como objetivo en los planes urbanos.

En suma, la planificación urbana actual se debería abrir a los planteamientos del desarrollo urbano sostenible, y a la realización de las buenas prácticas de la gestión inteligente (es decir, a la gobernabilidad); a fin de producir el perfeccionamiento de los sistemas y de los instrumentos del urbanismo, con el nuevo y mayor conocimiento de la cambiante e impredecible naturaleza de los comportamientos reales del desarrollo urbano, replanteados en muchos aspectos procedimentales y de gestión, liberados de su carga de voluntarismo y de su improcedente empeño de no contar con el mercado. Enriquecidos también por una mayor consideración de los problemas ambientales y por la asimilación de nuevas formas de pactar con la arquitectura ${ }^{7}$.

En este marco conceptual, se propone que la formulación del Plan de Desarrollo Metropolitano LimaCallao 2013-2035 se haga con el 
enfoque emergente de la planificación del desarrollo urbano sostenible, que debe entenderse como una interdisciplina orientada a prever, procurar, promover, orientar y regular la conjugación equilibrada de los siguientes componentes en los centros urbanos o ciudades: ${ }^{8}$

- La relación de la ciudad con su territorio de influencia, que está vinculada a la implementación de propuestas de ordenamiento territorial regional y de acondicionamiento territorial provincial.

- La competitividad urbana, que está vinculada a la implementación de propuestas de generación y difusión de ventajas competitivas en la ciudad y en su territorio circundante, a fin de atraer inversiones, desarrollar actividades económicas generadoras de riqueza y empleo, $\mathrm{y}$ ofrecer mejor calidad de vida a su población; en el contexto de un mundo globalizado.

- La funcionalidad urbana, que está vinculada a la implementación de propuestas de acondicionamiento urbano territorial, zonificación urbana, sistema vial urbano $\mathrm{y}$ equipamiento urbano.

- La calidad urbanística y paisajistica, que están vinculadas a la implementación de propuestas de mantenimiento, recuperación y/o acondicionamiento físico de espacios públicos, patrimonio urbanístico monumental, patrimonio arqueológico y del entorno de la ciudad.

- La sustentabilidad ambiental, que está vinculada a la implementación de propuestas de ordenamiento ambiental del medio ambiente natural y construido para aminorar los impactos ambientales negativos.

- La gestión de riesgos de desastres, que está vinculada a la prevención, reducción y seguimiento de los factores de riesgo de desastre, asî como a la adecuada preparación y respuesta ante situaciones de desastre.

- La equidad social urbana, que está vinculada a la implementación de propuestas de dotación y mejoramiento de servicios básicos, de acceso al suelo y programas de vivienda, y de renovación urbana para los diferentes estratos sociales en la ciudad, en función de estándares normativos.

- La identidad cultural, que está vinculada a la implementación de programas de educación vial, ciudadana, ambiental y de gestión de riesgo de desastres, cultura urbana y revaloración de la identidad cultural local.

- La gobernabilidad urbana (o gobernanza), que está vinculada a la implementación de propuestas de gestión urbana, modernización institucional municipal, y de promoción de mecanismos de concertación interinstitucional, de participación ciudadana, y de institucionalización, seguimiento $\mathrm{y}$ evaluación de planes.

Lima, 14 de Agosto del 2013. 


\section{NOTAS}

1 CPI (2012) "Perú: Población 2012”. Compañía Peruana de Estudios de Mercado y Opinión Pública SAC, Market Report Julio 2012. Lima, Perú.

2 FERNANDEZ GUELL, José Miguel (1997) "Planificación Estratégica de Ciudades". Págs. 52-53. Editorial Gustavo Gili. Barcelona, España.

3 DORICH, Luis (1996) "Al Rescate de Lima”. Colegio de Arquitectos del Perú CAP, Instituto de Urbanismo y Planificación del Perú - IUPP. Lima, Perú.

4 STEINBERG, Florián y CUBAS Carlos (2001) - "Planificación Estratégica Urbana”. PEGUP - Foro Ciudades para la Vida. Lima, Perú.

5 FERNANDEZ GUELL, José Miguel (1997) "Planificación Estratégica de Ciudades". Editorial Gustavo Gili. Barcelona, España.

6 CASTILLO, Rodolfo(2005)- "LaPlanificación Urbana del Área Metropolitana Lima Callao 1988 - 2004: ¿Causa Perdida o Reto Posible? Una Aproximación desde una
Perspectiva Emergente y Proactiva". Tesis de Magister en Asentamientos Humanos y Medio Ambiente. Instituto de Estudios Urbanos y Territoriales, Facultad de Arquitectura, Diseño y Estudios Urbanos, de la Pontifica Universidad Católica de Chile. Santiago, Chile.

7 DE TERAN, Fernando (1999) "Planeamiento Urbano Territorial en el Siglo XXI". Revista Urban No 4. Barcelona, España.

8 CASTILLO, Rodolfo (2012) - "La Planificación Urbana en el Perú 1947 2011: ¿Del Urbanismo Mágico a la Planificación del Desarrollo Urbano Sostenible? Apuntes Para Una Biografia y Una Propuesta". Tesis de Maestría en Planificación Urbana y Regional. Facultad de Arquitectura, Urbanismo y Artes, Sección de Postgrado y Segunda Especialización Profesional, Universidad Nacional de Ingeniería. Lima, Perú. 